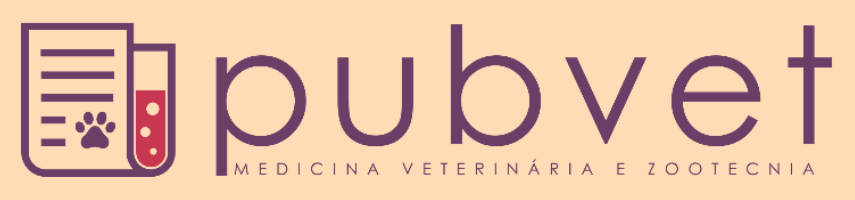

https://doi.org/10.31533/pubvet.v13n4a315.1-7

\title{
Frequência de Ancylostoma spp. em amostras fecais ambientais nas praças públicas de Pedro Osório, RS
}

Tainá Ança Evaristo ${ }^{* \bullet}$, Julia Somavilla $\operatorname{Lignon}^{1} \bullet$, Thaíssa Gomes Pellegrin' ${ }^{1}$, João Luis $\operatorname{Camassola}^{1}$, Alexsander Ferraz $^{2} \bullet$, Maysa Seibert Leão ${ }^{1}{ }^{\bullet}$, Gabriela Garcia Fuentes $^{1} \bullet$, Tatiana de Ávila Antunes $^{3}$, Felipe Geraldo Pappen ${ }^{4}$, Diego Moscarelli Pinto ${ }^{4} \bullet$

${ }^{I}$ Graduanda em Medicina Veterinária, Grupo de Estudos em Enfermidades Parasitárias. Universidade Federal de Pelotas, Pelotas, RS. ${ }^{2}$ Doutorando em Clínica de Pequenos Animais, Laboratório de Doenças Parasitárias. Universidade Federal de Pelotas, Pelotas, RS. ${ }^{3}$ Pós-graduanda em Zoonoses Parasitárias, Grupo de Estudos em Enfermidades Parasitárias. Universidade Federal de Pelotas, Pelotas, RS. ${ }^{4}$ Docente no Departamento de Medicina Veterinária Preventiva, Grupo de Estudos em Enfermidades Parasitárias. Universidade Federal de Pelotas, RS. Autor para correspondência, E-mail: evaristo.medvet@gmail.com

Resumo. Foram acompanhadas duas praças municipais na cidade de Pedro Osório/RS, entre os meses de dezembro de 2017 e novembro de 2018, sendo coletadas amostras fecais ambientais com a finalidade da identificação de parasitos com potencial zoonótico, como o Ancylostoma spp., potencial causador da dermatite parasitária denominada Larva Migrans Cutânea (LMC). O método diagnóstico utilizado foi a técnica copro-parasitológica Willis-Mollay. Obteve-se como resultado a prevalência de $61,11 \%$ e $50 \%$ de ovos de Ancylostoma spp., respectivamente. Os dados amostrais foram submetidos a análise estatística conforme Distribuição Normal Padrão (Z) e Intervalo de Confiança (IC) para proporção, com nível de significância de 5\% $(\alpha=1,96)$, obtendo-se com IC em P1 $(0,6178$ $\leq \pi \leq 0,6044)=95 \%$ e em P2 $(0,5136 \leq \pi \leq 0,4864)=95 \%$, demonstrando que há a possibilidade da população local contaminar-se com esse agente zoonótico no ambiente.

Palavras chave: zoonose, cães, saúde pública, parasitos gastrointestinais

\section{Frequency of Ancylostoma spp. in environmental fecal samples in the public squares of Pedro Osório, RS}

\begin{abstract}
Two municipal squares were monitored in the city of Pedro Osório / Two municipal squares were monitored in the city of Pedro Osório, RS, between December 2017 and November 2018, and environmental fecal samples were collected for the identification of parasites with potential zoonotic, such as Ancylostoma spp., a potential causative agent of parasitic dermatitis called Larva Migrans Cutanea (CML). The diagnostic method used was the copro-parasitological technique Willis-Mollay. The prevalence of $61.11 \%$ and $50 \%$ of Ancylostoma spp. Eggs, respectively, was obtained as a result. The sample data were submitted to statistical analysis according to Standard Normal Distribution (Z) and Confidence Interval (CI) for proportion, with a significance level of 5\% $(\alpha=1.96)$, obtained with CI at P1 $(0,6178 \leq \leq 0.6044)=95 \%$, and in $\mathrm{P} 2(0.5136 \leq \leq 0.4864)=95 \%$, demonstrating that there is a possibility that the local population may become contaminated with this zoonotic agent in the environment.
\end{abstract}

Keywords: zoonosis, dogs, public health, gastrointestinal parasites 


\title{
Frecuencia de Ancylostoma spp. en muestras fecales ambientales en las plazas públicas Pedro Osório, RS
}

\begin{abstract}
Resumen. En la ciudad de Pedro Osório / RS, entre los meses de diciembre de 2017 y noviembre de 2018, se analizaron dos plazas municipales, siendo recogidas muestras fecales ambientales con la finalidad de la identificación de parásitos con potencial zoonótico, como el Ancylostoma spp., potencial causante de la dermatitis parasitaria denominada Larva Migrans Cutánea (LMC). El método diagnóstico utilizado fue la técnica copro-parasitológica Willis-Mollay. Se obtuvo como resultado la prevalencia de $61,11 \%$ y 50\% de huevos de Ancylostoma spp., Respectivamente. Los datos muestrales se sometieron a análisis estadístico según la distribución normal estándar $(Z)$ y el intervalo de confianza (IC) para la proporción, con un nivel de significancia del 5\% $(\alpha=1,96)$, obteniéndose con IC en P1 $(0,6178 \leq \pi \leq 0,6044)=95 \%$ e em P $2(0,5136 \leq \pi \leq 0,4864)=95 \%$, demostrando que hay la posibilidad de la población local contaminarse con ese agente zoonótico en el ambiente.
\end{abstract}

Palabras clave: zoonosis, perros, salud pública, parásitos gastrointestinales

\section{Introdução}

Os animais de companhia, principalmente cães, são cada vez mais importantes na sociedade em todo mundo, sendo muitas vezes considerados companheiros nos núcleos familiares, contribuindo para o desenvolvimento físico, social, emocional e do bem-estar dos seus tutores, de crianças e de idosos. No entanto, também podem carretam maior exposição humana a agentes zoonóticos (Dal-Farra, 2003; Gennari et al., 1999; Jennings, 1997; Raina et al., 1999). O número elevado de cães errantes portadores de parasitos intestinais e com acesso direto a locais de lazer, como praças públicas e praias, expõe os frequentadores a infecções provocadas por contato direto ou indireto com fezes contaminadas por agentes de característica zoonótica (Santarém et al., 2004; Scaini et al., 2003).

Ancylostoma brasilienses e Ancylostoma caninum são HD de cães e gatos, gerando quadro de anemia acentuada pela espoliação do parasito no intestino delgado, denominando-se Ancilostomose canina, semelhante ao que ocorre no ser humano quando é parasitado por Necator americanus ou Ancylostoma duodenale (Ferreira, 2012). Os ancilostomídeos são parasitos que albergam o intestino delgado do ser humano, onde dois espécimes são amplamente distribuídos, sendo o Necator americanus e o Ancylostoma duodenale, responsáveis por uma anemia pronunciada no seu hospedeiro definitivo (HD) (Bowman, 2010; Taylor et al., 2017).

As larvas filarióides de A. brasilienses ou A. caninum quando penetram ativamente na pele do seu hospedeiro acidental (HA), ou ser humano, causa a síndrome conhecida como Larva Migrans Cutânea (LMC.), popularmente denominada "bicho geográfico". As larvas de estádio L3 não completam seu ciclo por não estarem em um hospedeiro preferencial, abrindo apenas ductos entre a epiderme e a derme, até serem eliminadas pelo sistema imune do indivíduo, ou por tratamento (Bowman, 2010; Taylor et al., 2017).

O presente estudo teve como objetivo verificar a frequência do nematelminto gastrintestinal Ancylostoma spp. em amostras fecais ambientais coletadas nas praças públicas do município de Pedro Osório, Rio Grande do Sul, Brasil.

\section{Material e métodos}

O município de Pedro Osório localiza-se no Sul do Rio Grande do Sul, latitude $31^{\circ} 51^{\prime}$ '51' $\mathrm{S}$ e longitude $52^{\circ} 49^{\prime} 24^{\prime}$ ' W, com 7.755 habitantes, área territorial de $608,789 \mathrm{~km}^{2}$, densidade demográfica de $12,83 \mathrm{hab} / \mathrm{km}^{2}$, IDHM (Índice de Desenvolvimento Humano) de 0,678, tendo apenas 40,2\% de esgotamento sanitário adequado, dados obtidos pelo IBGE em 2018. 
Foram coletadas amostras fecais ambientais provenientes de animais de companhia localizadas em duas praças centrais públicas municipais (P1 e P2) em Pedro Osório, Rio Grande do Sul, entre os meses de dezembro de 2017 até novembro de 2018, totalizando 12 meses do estudo epidemiológico. Foram coletadas seis amostras ambientais por mês de cada local, totalizando 72 amostras fecais $\left(n^{1}=72 ; n^{2}=\right.$ 72) em cada praça, acumulando-se $n^{(\mathrm{t})}=144$ amostras. As fezes foram retiradas do ambiente utilizando luvas de procedimento para manter a integridade do pesquisador, bem como foram mantidas em sacolas plásticas devidamente identificadas conforme o mês e o local, armazenadas em caixas isotérmicas com gelo retornável, sendo encaminhadas para posterior processamento no Grupo de Estudos em Enfermidades Parasitárias (GEEP), na Faculdade de Veterinária (FaVet), Universidade Federal de Pelotas (UFPel).

Para o diagnóstico laboratorial dos nematelmintos foi utilizada a técnica copro-parasitológica de Willis (1921) que se baseia no princípio de flutuação em solução hiper saturada de glicose, que desta forma faz com que os ovos de característica leve, como dos nematoides, flutuem em relação à diferença de densidade imposta pela solução inserida na técnica. Para elaboração do método de Willis (1921) separou-se até $5 \mathrm{~g}$ de fezes da amostra e homogeneizou-se a mesma com $20 \mathrm{ml}$ de solução hiper saturada, após a amostra foi passada em um Tamis e inseriu-se o restante do conteúdo em um tubo de ensaio. Colocou-se uma lamínula de $18 \times 18$ sobre o menisco formado com a solução e após 20 minutos foi realizada leitura em microscopia óptica com aumento de 10x. Os ovos do parasita foram identificados conforme sua característica morfo-tintorial.

Após planilhar os resultados obtidos, os dados foram submetidos à análise de média amostral $(x)$ variância $\left(\mathrm{S}^{2}\right)$ e desvio padrão (S), bem como estabeleceu-se o intervalo de confiança (IC) para a proporção $(\pi)$ dos dados conforme a média amostral $(x)$ para variável aleatória discreta, seguindo Distribuição de Probabilidade Normal Padrão (Z), com nível de significância de 5\% $(\alpha=1,96)$.

\section{Resultados}

Tabela 1. Frequência $\left(n^{1}\right)$ de ovos de Ancylostoma spp. em amostras fecais ambientais recolhidas de uma praça pública municipal (P1) em Pedro Osório, RS

\begin{tabular}{lccccc}
\hline Meses/ano & $\mathrm{N}^{\mathrm{o}}$ de amostras & $\mathrm{fi}$ & $\mathrm{Fi}$ & $\mathrm{fi}(\%)$ & $\mathrm{Fi}(\%)$ \\
\hline Dezembro/17 & 6 & 3 & 3 & 0,0681 & 0,0681 \\
Janeiro/18 & 6 & 6 & 9 & 0,1363 & 0,2044 \\
Fevereiro/18 & 6 & 6 & 15 & 0,1363 & 0,3407 \\
Março/18 & 6 & 6 & 21 & 0,1363 & 0,477 \\
Abril/18 & 6 & 4 & 25 & 0,0909 & 0,5679 \\
Maio/18 & 6 & 3 & 28 & 0,0681 & 0,636 \\
Junho/18 & 6 & 3 & 31 & 0,0681 & 0,7041 \\
Julho/18 & 6 & 0 & 31 & 0,0909 & 0,7041 \\
Agosto/18 & 6 & 4 & 35 & 0,0454 & 0,795 \\
Setembro/18 & 6 & 2 & 40 & 0,0681 & 0,9085 \\
Outubro/18 & 6 & 3 & 0,0909 & $\approx 1$ \\
Novembro/18 & 6 & 4 & - & $\approx 1$ & - \\
Total $\left(\sum\right)$ & 72 & 44 & 44 & \\
\hline
\end{tabular}

Legenda: f i - frequência absoluta de amostras positivas para Ancylostoma spp.; Fi - frequência absoluta acumulada de amostras positivas para Ancylostoma spp.; Fi (\%) - frequência absoluta acumulada de amostras positivas em porcentagem para Ancylostoma spp.; fi (\%) - frequência absoluta de amostras positivas em porcentagem para Ancylostoma spp; 17 - referente ao ano de 2017; 18 - referente ao ano de 2018; $\approx$ - fator de arredondamento.

Conforme o $n$ amostral total $\left(n^{t}\right)$, ou seja, 144 amostras estabeleceram-se a média $(x)$, variância $\left(\mathrm{S}^{2}\right)$ e desvio padrão (S) para os resultados obtidos através da prevalência de ovos de Ancylostoma spp. na Praça $1\left(\mathrm{P} 1 ; n^{1}=72\right)$ e Praça $2\left(\mathrm{P} 2 ; n^{2}=72\right)$, para a partir destes, utilizando a Distribuição de Probabilidade Normal Padrão (Z), estabeleceu-se conforme a variável discreta, o intervalo de confiança (IC) para proporção, com nível de significância de 5\%, tendo $\alpha=1,96$. 
Tabela 2. Frequência $\left(n^{2}\right)$ de ovos de Ancylostoma spp. em amostras fecais ambientais recolhidas de uma praça pública municipal (P2) em Pedro Osório, RS

\begin{tabular}{lccccc}
\hline \multicolumn{1}{c}{ Meses/ano } & $\mathrm{N}^{\mathrm{o}}$ de amostras & $\mathrm{fi}$ & $\mathrm{Fi}$ & $\mathrm{fi}(\%)$ & $\mathrm{Fi}(\%)$ \\
\hline Dezembro/17 & 6 & 2 & 2 & 0,0555 & 0,0555 \\
Janeiro/18 & 6 & 4 & 6 & 0,1111 & 0,0166 \\
Fevereiro/18 & 6 & 2 & 8 & 0,0555 & 0,2221 \\
Março/18 & 6 & 4 & 12 & 0,1111 & 0,3332 \\
Abril/18 & 6 & 4 & 16 & 0,1111 & 0,4443 \\
Maio/18 & 6 & 3 & 19 & 0,0833 & 0,5276 \\
Junho/18 & 6 & 3 & 22 & 0,0833 & 0,6109 \\
Julho/18 & 6 & 0 & 22 & 0,1111 & 0,6109 \\
Agosto/18 & 6 & 4 & 30 & 0,1111 & 0,722 \\
Setembro/18 & 6 & 4 & 33 & 0,0833 & 0,8331 \\
Outubro/18 & 6 & 3 & 0,0833 & 0,9164 \\
Novembro/18 & 6 & 3 & - & $\approx 1$ & $\approx 1$ \\
Total $\left(\sum\right)$ & 72 & 36 & 36 & - \\
\hline
\end{tabular}

Legenda: $\mathrm{f}$ i - frequência absoluta de amostras positivas para Ancylostoma spp.; Fi - frequência absoluta acumulada de amostras positivas para Ancylostoma spp.; Fi (\%) - frequência absoluta acumulada de amostras positivas em porcentagem para Ancylostoma spp.; fi (\%) - frequência absoluta de amostras positivas em porcentagem para Ancylostoma spp; 17 - referente ao ano de 2017; 18 - referente ao ano de 2018; $\approx$ - fator de arredondamento.

Seguindo Distribuição Normal Padrão (Z), estabeleceu o Intervalo de Confiança (IC) por proporção $(\pi)$, com nível de significância de $5 \%(\alpha=1,96)$, para a porcentagem de ocorrência de número de amostras positivas para o gênero Ancylostoma spp. na Praça 1, P1 $(0,6178 \leq \pi \leq 0,6044)=95 \%$, e da mesma forma também para Praça $2, \mathrm{P} 2(0,5136 \leq \pi \leq 0,4864)=95 \%$. O erro da taxa de ocorrência de ovos de Ancylostoma spp. para este estudo é de 0,0067 e 0,0136 - P1 e P2.

Tabela 3. Média, variância e desvio padrão da prevalência de ovos de Ancylostoma spp. em amostras fecais ambientais em duas praças de Pedro Osório, RS.

\begin{tabular}{lcc}
\hline & Praça 1 (P1) & Praça 2 (P2) \\
\hline$x(\%)$ & 61,1111 & 50 \\
$\mathrm{~S}^{2}$ & 1,2111 & 1,2816 \\
$\mathrm{~S}$ & 1,1050 & 1,1320 \\
\hline
\end{tabular}

Legenda: $x(\%)$ - média amostral em porcentagem; $\mathrm{S}^{2}$ - variância amostral; $\mathrm{S}$ - desvio padrão amostral.

\section{Discussão}

A Larva Migrans Cutânea é considerada uma doença endêmica em países tropicais e subtropicais, como países asiático, africanos e latinos (Macias et al., 2013). Sendo descrita pela primeira vez em 1874, porém só x 50 anos depois foi realizada a primeira biópsia cutânea, onde foi comprovada a presença de um parasita nematoide que penetrava de forma ativa na pele e migrava através da mesma (Ferreira et al., 2003; Heukelbach \& Feldmeier, 2008). A parasitose é pouco comum em países industrializados, apesar de ser a segunda maior causa de morbidades nos turistas, seguida da diarreia (Moreira, 2006). O seu diagnóstico é clínico, baseado na história clínica do hospedeiro acidental, mas também nas lesões tipicamente cutânea.

A técnica abordada neste estudo para identificação de ovos de Ancylostoma spp. foi o método de Willis (1921) que corrobora com o que cita a literatura, caracterizando estes helmintos por terem ovos leves, elipsoides, com dimensões entre 55 e $77 \mu \mathrm{m}$, de comprimento por 34 a $45 \mu \mathrm{m}$ de largura, membrana fina e lisa, com mórulas compostas por células de formato globoso em seu interior, facilitando assim sua flutuação (Marchioro et al., 2013; Marder et al., 2004).

Na cidade de Cáceres, extremo norte do Pantanal foram coletadas 120 amostras fecais do perímetro urbano em quatro bairros: Cohab Nova, Rodeio, Comunidade Carne Seca e margue esquerda do Rio Paraguai, pelo método de Hoffman, Pons e Janer e Faust foram detectadas 34,16\% de ovos característicos da família Ancylostomidae. O resultado é inferior ao encontrado nas 144 amostras no 
município de Pedro Osório, com média amostral de ocorrência de 61,11\% - P1 e 50\% - P2 (Lima Rosales $\&$ Malheiros, 2017). Em estudo realizado por Prestes et al. (2015) foram coletadas amostras de solo de praças de municípios do Sul do Rio Grande do Sul, sendo seis municípios, entre eles Pedro Osório. Foram constatados ovos de Toxocara spp., Ascaris spp., Trichuris spp. e superfamília Strongyloidea, porém, neste estudo não foi evidenciado a presença de Ancylostoma spp. Na pesquisa estabelecida por Prestes et al. (2015) foi constata a presença de animais de companhia, fezes e ausência de cercas nas praças, o que corroboram com o presente estudo. A presença de barreira ou cercas que impeçam o livre acesso de cães e gatos às praças são constituintes que podem prevenir a presença de parasitos com potencialidade zoonótica nestes locais (Sprenger et al., 2014).

Em estudo na cidade de Volta Redonda, em 120 amostras fecais recolhidas nas praças da cidade em 20 pontos diferentes, a taxa de ocorrência de Ancylostoma spp. foi de 29,4\%, inferior ao intervalo de confiança sob proporção que foi estabelecido para a ocorrência deste nematoide em ambas praças no município de Pedro Osório, sendo P1 $(0,6178 \leq \pi \leq 0,6044)$ e P2 $(0,5136 \leq \pi \leq 0,4864)$, respectivamente (Stutz et al., 2017). Avaliando a prevalência e os gêneros parasitários em cães domiciliados do município de Dois Vizinhos/Paraná, foram coletadas amostras coproparasitológicas de 123 cães e, destes, 17 albergavam Ancylostoma, o que ocorre em várias localidades do Brasil, tendo este gênero como a espécime mais frequentemente encontrado em cães, causando nos animais deficiência de ferro, anemia microcítica hipocrômica, apatia e perda de peso, dado a característica de hematofagia do parasito (Katagiri \& Oliveira-Sequeira, 2007; Martins et al., 2012). Além disso, a eliminação de fezes de cães previamente parasitados no solo favorece a transmissão da síndrome Larva Migrans Cutânea (LMC) causada por formas larvais de terceiro estádio de ancilostomídeos, viáveis no solo, principalmente em crianças que têm hábitos de brincar em terra e areia, constituindo importante problema de saúde pública.

$\mathrm{Na}$ região Centro-Oeste do Brasil são observadas prevalência que giram ao redor 84,4\% de positividade para helmintos da família Ancylostomidae (Almeida et al., 2007) devido a característica do clima, o que diverge do presente estudo, com prevalência de $61,1 \%$ e $50,0 \%$ para praças estudadas - P1 e P2. A elevada ocorrência de Ancylostoma spp. pode estar relacionada ao ciclo de vida destes parasitas, que infectam seus hospedeiros definitivos, como cães, por penetração ativa na pele, ingestão fecal-oral, além de transmissão transplacentária e transmamária, estes que estão diretamente com contato ao solo (Alves et al., 2016).

No município de Pedro Osório, de janeiro de 2017 a janeiro de 2018 em praças centrais, Evaristo et al. (2018) detectaram por análises coproparasitológicas 60 amostras positivas para Ancylostoma spp. de 72 , o que difere do resultado encontrado em no atual período de estudo no município, sendo 44 e 36 amostras positivas para cada 72 analisadas por praça.

Segundo Fortes (2004), a temperatura ideal para o desenvolvimento das formas pré-parasitárias de Ancylostoma spp. está entre $23^{\circ} \mathrm{C}$ a $30^{\circ} \mathrm{C}$, diferentemente ao que ocorre como média de temperatura em Pedro Osório/RS, sendo de $10,8^{\circ} \mathrm{C}$ ao ano, onde janeiro geralmente é o mês mais quente $\left(23,7^{\circ} \mathrm{C}\right)$, o que pode explicar a contaminação em 100\% das amostras coletadas na Praça 1 em janeiro, fevereiro e março de 2018.

Existe divergência quanto à prevalência de geohelmintos ao redor do mundo, atribuindo-se a diferentes condições ambientais, climáticas e epidemiológicas, que podem estar ligadas a fatores socioeconômicos e culturais de cada região, unificando-se as diversidades de técnicas laboratoriais e métodos de coleta e armazenamento de amostras de fezes e areia utilizados em cada estudo (Bojanich et al., 2015).

\section{Conclusão}

De acordo com os resultados obtidos no presente estudo, verificou-se que Ancylostoma spp., é um parasito frequente nas praças públicas analisadas em Pedro Osório, Rio Grande do Sul, representando um risco à saúde humana e animal. 


\section{Referências bibliográficas}

Almeida, A. B. P., Sousa, V. R. F., Dalcin, L. \& Silva Justino, C. H. (2007). Contaminação por fezes caninas das praças públicas de Cuiabá, Mato Grosso. Brazilian Journal of Veterinary Research and Animal Science, 44(2):132-136.

Alves, A. P. d. S. M., Coelho, M. D. G., Santos, I. A., Bozo, L. S. O. \& Maciel, L. T. R. (2016). Contaminação em logradouros do Município de Pindamonhangaba-SP, por parasitos potencialmente zoonóticos em fezes caninas. Revista Ciência e Saúde, 1(1):45-50.

Bojanich, M. V., Alonso, J. M., Caraballo, N. A., Itatí Schöller, M., López, M. d. 1. Á., García, L. M. \& Basualdo, J. Á. (2015). Assessment of the presence of Toxocara eggs in soils of an arid area in central-western Argentina. Revista do Instituto de Medicina Tropical de São Paulo, 57(1):73-76.

Bowman, D. D. (2010). Parasitologia veterinária: Elsevier.

Dal-Farra, R. A. (2003). Representações de animais de companhia na cultural contemporânea: uma análise na mídia impressa. Semiosfera, 3(7).

Evaristo, T. A., Ferraz, A., Pires, B. S., Martins, N. S., Antunes, T. Á. \& Pinto, D. M. (2018). Prevalência de parasitos gastrintestinais em amostras fecais de cães em praças públicas nos municípios de Pedro Osório e Cerrito, RS. Atas de Saúde Ambiental, 671-84.

Ferreira, C., Machado, S. \& Selores, M. (2003). Larva migrans cutânea em idade pediátrica: a propósito de um caso clínico. Nascer e Crescer, 12261-264.

Ferreira, M. U. 2012. Parasitologia Contemporânea. Guanabara Koogan.

Fortes, E. (2004). Parasitologia veterinária (4 ed.). São Paulo: Editora ícone.

Gennari, S. M., Kasai, N., Pena, H. F. d. J. \& Cortez, A. (1999). Occurrence of protozoa and helminths in faecal samples of dogs and cats from São Paulo city. Brazilian Journal of Veterinary Research and Animal Science, 36(2):1-4.

Heukelbach, J. \& Feldmeier, H. (2008). Epidemiological and clinical characteristics of hookwormrelated cutaneous larva migrans. The Lancet Infectious Diseases, 8(5):302-309.

Jennings, L. B. (1997). Potential benefits of pet ownership in health promotion. Journal of Holistic Nursing, 15(4):358-372.

Katagiri, S. \& Oliveira-Sequeira, T. C. G. (2007). Zoonoses causadas por parasitas intestinais de cães e o problema do diagnóstico. Arquivos do Instituto Biologico, 74(2):175-184.

Lima Rosales, T. F. \& Malheiros, A. F. (2017). Contaminação Ambiental por enteroparasitas presentes em fezes de cães em uma região do Pantanal. O Mundo da Saúde, 4(3):368-377.

Macias, V. C., Carvalho, R., Chaveiro, A. \& Cardoso, J. (2013). Larva Migrans cutânea-a propósito de um caso clínico. Revista da Sociedade Portuguesa de Dermatologia e Venereologia, 71(1):93-96.

Marchioro, A., Colli, C., Ferreira, É., Tiyo, R., Mattia, S., Souza, W. \& Falavigna-Guilherme, A. (2013). Identification of public areas with potential toxocariasis transmission risk using Geographical Information Systems. Acta Parasitologica, 58(3):328-333.

Marder, G., Ulon, S. N., Bottinelli, O. R., Fleitas, Z. M., Lotero, D. A., Ruiz, R. \& Arzú, R. A. (2004). Infestación parasitaria en suelos y materia fecal de perros y gatos de la ciudad de Corrientes. Revista Veterinaria, 15(2):70-72.

Martins, C. M., Barros, C. d. C. d., Bier, D., Marinho, A. P., Figueiredo, J. M. G., Hoffmann, J. L., \& Biondo, A. W. (2012). Dog parasite incidence and risk factors, from sampling after one-year interval, in Pinhais, Brazil. Revista Brasileira de Parasitologia Veterinária, 21(2):101-106.

Moreira, H. (2006). Emigração Portuguesa (Estatísticas retrospectivas e reflexões temáticas). Revista de Estudos Demográficos, 3847-65.

Prestes, L. F., Jeske, S., Santos, C. V., Gallo, M. C. \& Villela, M. M. (2015). Contaminação do solo por geohelmintos em áreas públicas de recreação em municípios do sul do Rio Grande do Sul (RS), Brasil. Revista de Patologia Tropical/Journal of Tropical Pathology, 44(2):155-162.

Raina, P., Waltner-Toews, D., Bonnett, B., Woodward, C. \& Abernathy, T. (1999). Influence of companion animals on the physical and psychological health of older people: An analysis of a oneyear longitudinal study. Journal of the American Geriatrics Society, 47(3):323-329. 
Santarém, V. A., Giuffrida, R. \& Zanin, G. A. (2004). Larva migrans cutânea: ocorrência de casos humanos e identificação de larvas de Ancylostoma spp em parque público do município de Taciba, São Paulo. Revista Brasileira de Medicina Tropical, 37(2):179-181.

Scaini, C. J., Toledo, R. N., Lovatel, R. M., Dionello, M. A., Gatti, F. A. A., Susin, L. R. O. \& Signorini, V. R. M. (2003). Contaminação ambiental por ovos e larvas de helmintos em fezes de cães na área central do Balneário Cassino, Rio Grande do Sul. Revista da Sociedade Brasileira de Medicina Tropical, 36(5):617-619.

Sprenger, L. K., Green, K. T. \& Molento, M. B. (2014). Geohelminth contamination of public areas and epidemiological risk factors in Curitiba, Brazil. Revista Brasileira de Parasitologia Veterinária, 23(1):69-73.

Stutz, E. T. G., Souza, A. P., Santos Lima, I. \& Guimarães, P. M. S. (2017). Ocorrência de Ancylostoma sp. em fezes de animais coletados em praças e parques públicos do município de Volta Redonda. Episteme Transversalis, 5(2).

Taylor, M. A., Coop, R. L. \& Wall, R. L. (2017). Parasitologia Veterinária. Rio de Janeiro, Brasil: Guanabara Koogan.

Willis, H. H. (1921). A simple levitation method for the detection of hookworm ova. Medical Journal of Australia, 2(18):375-376.

Recebido: 12 março, 2019

Aprovado: 1 abril, 2019

Publicado: 30 abril, 2019.

Licenciamento: Este artigo é publicado na modalidade Acesso Aberto sob a licença Creative Commons Atribuição 4.0 (CC-BY 4.0), a qual permite uso irrestrito, distribuição, reprodução em qualquer meio, desde que o autor e a fonte sejam devidamente creditado 